\title{
AN AUTOMATED SEARCH FOR LOW SURFACE BRIGHTNESS DWARF ELLIPTICAL GALAXIES
}

\section{Z. MORSHIDI, R.M. SMITH AND J.I. DAVIES}

Department of Physics \& Astronomy, University of Wales, College of Cardiff, P.O.Box 913, Cardiff CF2 3YB, UK

A new automated technique applied to APM scans of UKSTU photographic plates has been used to search for LSBGs in the Fornax cluster. 92 galaxies, almost all of which were classified by Ferguson (1989) as dwarf ellipticals, were found. Fields to the East and West of the cluster were found to have 46 and 13 LSBGs, respectively, as shown in the figure below ( $\square$ are LSBGs, $\boldsymbol{\Delta}$ are brighter galaxies).

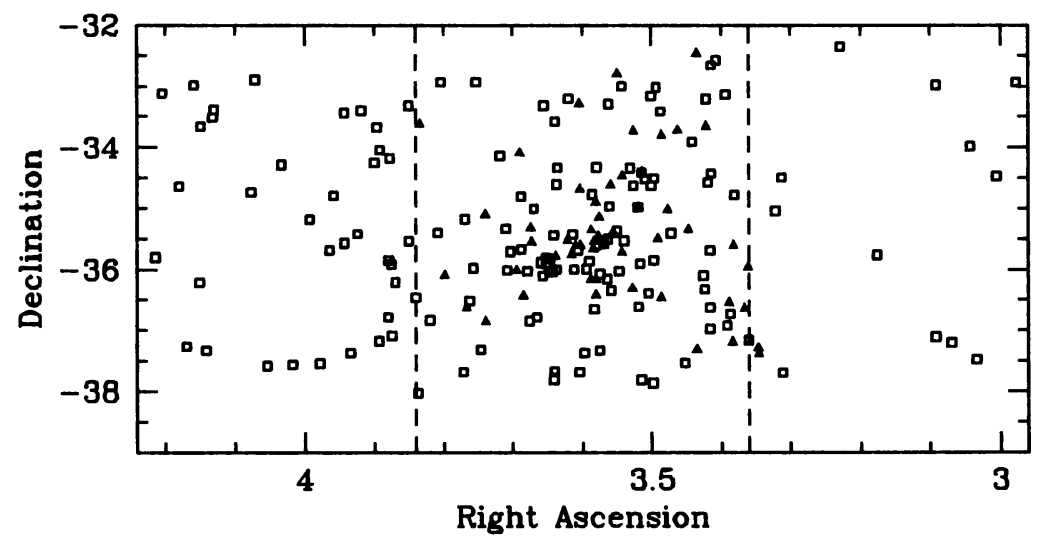

The LSBGs are clustered weakly towards the Fornax plate, in confirmation of the suggestion of Davies (1989). An estimate of the clustering size of the LSBGs indicates that they have a scale length approximately 5 times that of the brighter galaxies.

\section{References}

Davies, J.I., 1989, Ph.D. Thesis, University of Wales College of Cardiff Ferguson, H.C., 1989, Astron. J., 98, 367 